\title{
Coherencia EN EL DERECHO: CONSERVADURISMO Y FIDELIDAD A LA BASE DE REGLAS
}

Juliano S.A. Maranhão*

Traducción de Pablo Ariel Rapetti* *

* University of São Paulo Law School.

** Universidad Nacional del Sur

\section{1 - INTRODUCCIÓN}

El de la coherencia ha sido un tema candente en la teoría del derecho. En parte, creo, por el calor heredado del ataque de Dworkin al positivismo (Dworkin, 1986), que puede ser interpretado como un ataque al fundacionalismo jurídico por parte de una teoría coherentista global (el derecho como integridad). Esta última identifica a las proposiciones sobre el derecho como justificadas si son el producto de un ajuste argumentativo e interpretativo entre el contenido de estándares morales y el contenido del material jurídico disponible (leyes y precedentes). El primero identifica como justificadas a las proposiciones sobre el derecho si se siguen de reglas 
básicas y, en última instancia, de una regla fundamental cuyo contenido no necesita justificación. Si bien han aparecido varias teorías coherentistas de este espíritu anti-positivista y las mismas han sido contraatacadas, poco se ha hecho por asentar sus conceptos, terminología y tesis principales, y así proveer una visión más clara de lo que significaría exactamente, dentro del campo del derecho, una teoría coherentista por oposición a una fundacionalista. No es sorprendente que muchos malentendidos se hayan producido, y ni siquiera es claro que la teoría de la integridad de Dworkin sea coherentista después de todo (Raz, 1994).

Creo que el trabajo de Amaya (Amaya, 2006) ha llenado esta laguna al proveer un completo y actualizado examen sobre el debate en epistemología en general y sobre las teorías jurídicas coherentistas. También ofrece mapas claros entre la discusión y los conceptos del campo de la epistemología y el de la justificación del derecho, tanto en el razonamiento probatorio como en el razonamiento normativo (interpretación jurídica). El trabajo sometido a esta discusión es sólo una mirada superficial a su tesis doctoral. Por esa razón, es muy conciso y no hace explícitos todos los fundamentos de sus diez proposiciones. Por ello, creo que esas proposiciones se ven debilitadas y no parecen demasiado controversiales (e.g. la primera tesis dice que la coherencia puede ser entendida en términos de satisfacción de restricciones, pero ella ve este modelo como el mejor para entender la coherencia).

En tan exhaustiva investigación de la materia, Amaya no pudo evitar hacer un balance y señalar qué modelos y rasgos de las diferentes teorías parecen capturar mejor el concepto de coherencia, particularmente en el derecho. Sus preferencias están expresadas en sus diez proposiciones, que básicamente comprenden (i) una defensa de la coherencia en el derecho como un valor y de las teorías coherentistas como alternativas plausibles e importantes tanto al escepticismo como al formalismo (que ella identifica con los modelos deductivos de ciencia jurídica), (ii) una visión de la coherencia como explicativa, basada en la relación medios-fines, posiblemente local (en lugar de necesariamente relacionadora de todas las oraciones de un conjunto de creencias) y contextual (los estándares de evaluación de la coherencia pueden variar de acuerdo al contexto), (iii) una preferencia por el modelo de 
satisfacción de restricciones de Thagard para representar a la coherencia, así como por el modelo de Olsson para representar cambios en el conjunto de creencias o normas para restaurar o incrementar la coherencia, (iv) una visión de la epistemología jurídica como justificación personal que conduce a quien realiza un razonamiento jurídico a la responsabilidad epistémica de manejar virtuosamente los estándares coherentistas de justificación.

En general estoy de acuerdo con sus afirmaciones, particularmente con su visión de la coherencia y la epistemología jurídica (ítems ii a iv) aún cuando no comparto exactamente sus preferencias por los modelos lógicos para representarla. Siento que estamos en desacuerdo acerca del rol que cumple la coherencia en el conocimiento del derecho (i). Creo que nuestro desacuerdo es una cuestión de grado con respecto al conservadurismo frente a las bases de una clausura coherentista del sistema normativo (o de nuestro conocimiento sobre el sistema normativo). Pero espero que la discusión aquí ayude a clarificar nuestra divergencia o muestre una convergencia. En mis comentarios, en vez de objetar tal o cual punto, aceptaré la invitación provista por la gran claridad de su exposición del campo de batalla de las posibles tesis sobre la coherencia en el derecho y trataré de desarrollar dos puntos, ambos relacionados con el razonamiento acerca de normas:

a) ¿Qué sería la base explicativa de la coherencia en el derecho y cuán conservadores deberíamos ser respecto de ella?

b) ¿Cuáles son las operaciones lógicas en la explicación constructiva de la base?

Entiendo a éstas como preguntas centrales de un proyecto más amplio: la búsqueda de una definición de una clausura coherentista de un sistema jurídico. La concepción del derecho como un sistema de normas clausurado por la relación de consecuencia lógica se ha mostrado muy fructífera pero insuficiente para dar cuenta de la complejidad del razonamiento jurídico. Alchourrón y Bulygin, quienes han trabajado esta concepción hasta una clara y precisa definición de un sistema jurídico 
lógicamente clausurado (Alchourrón y Bulygin, 1971), han luego concedido que quizás no todas las consecuencias lógicas de la base son derecho válido. La indagación en las razones objetivas de las reglas (Bulygin) o en la actitud disposicional del creador de derecho (Alchourrón, 1996) puede derrotar algunas de las consecuencias que son inconsistentes con aquellas razones o intenciones subjetivas. De ahí que la clausura lógica no sea suficiente para determinar qué es el sistema jurídico a partir del material dado por los textos jurídicos. La coherencia de sus reglas con sus propios fines es también relevante. El razonamiento basado en estos fines puede tener el efecto de suprimir algunas consecuencias normativas del sistema original, así como de añadir nuevas soluciones. Si lo que está en juego es la coherencia explicativa, esto significa que los elementos del sistema explican o son explicados por los otros ítems. Y la adición o eliminación de estos elementos dependerá de su capacidad de incrementar o de reducir los nexos explicativos del sistema. Entonces, para definir la clausura coherentista de un sistema jurídico es importante indagar primero, en qué podría consistir la base preliminar a ser explicada en el derecho y qué inferencias se ven involucradas en su expansión o contracción.

Creo que nuestra divergencia (o convergencia) se hará manifiesta al bosquejar estos puntos.

\section{LA BASE EXPLICATIVA DE LA JUSTIFICACIÓN JURÍDICA}

Años de disputa entre fundacionalistas y coherentistas en epistemología han producido gradualmente, si no una convergencia, un debilitamiento de sus tesis principales. Los fundacionalistas han aceptado la idea de que las creencias básicas (aquellas que no necesitan justificación y que justifican a todas las demás dentro de un sistema linear de creencias) pueden ser corregibles y que las razones de coherencia pueden jugar un rol en la justificación. En particular, si las creencias básicas son corregibles, debe haber una selección de creencias básicas (que no pueden ser justificadas por otras incorregibles). Si esto es cierto, la selección debería estar guiada por algún sistema de evaluaciones que determine cuál de nuestras creencias es más 
conducente a la verdad. Esto podría, en sí mismo, llevar a una selección coherentista de creencias básicas (Lehrer, 2000: 95). Los coherentistas, a su turno, han sido más flexibles con respecto a la pretensión de holismo (aquella de que nuestras creencias están justificadas en términos de una relación global de apoyo mutuo) y han aceptado tanto una dimensión local de coherencia como también la idea de que algunas de nuestras creencias puede tener un estatus privilegiado o de que se puede arribar psicológicamente a ellas sin apoyo en otras creencias (a pesar de que todos ellos insisten en que ninguna creencia podría permanecer en nuestro sistema de conocimiento sin referencia a otras creencias). Esto es particularmente cierto respecto de la coherencia explicativa, donde debe haber un punto de partida consistente en un conjunto de creencias a ser explicado.

Entonces, si uno es un fundacionalista soft o un coherentista soft el edificio de conocimiento debería ser construido a partir de una base preliminar de creencias que son "inocentes hasta que se demuestre lo contrario" (cf. Reid, 1985, apud Lehrer, p. 73). Éstas son de alguna manera privilegiadas, sea porque están enraizadas en nuestras prácticas de modo que su cuestionamiento es visto como irrazonable (aunque puedan ser equivocadas), sea porque nuestro sistema de evaluaciones nos provee de una competencia inmediata para identificarlas como las que más probablemente sean verdaderas. En el debate en epistemología estas creencias son representadas por esas evidencias perceptivas o verdades analíticas (al menos oraciones que sería lógicamente imposible que el agente creyera y que sean falsas). Por ejemplo, dentro del modelo de Thagard de satisfacción de restricciones, que Amaya suscribe, las proposiciones obtenidas por la observación tienen un grado adecuado de aceptación mientras que las otras dependen de los nexos de activación, positivos o negativos, que mantengan con el conjunto de proposiciones aceptadas.

Mi pregunta entonces es: ¿qué es exactamente lo que ocuparía el rol de "proposiciones observacionales" en la interpretación jurídica? ¿Cuáles son las evidencias inmediatas de la juridicidad o de que una cierta acción es jurídicamente correcta o incorrecta, en un conjunto de circunstancias particular? 
No nos comprometamos con el coherentismo en el punto de partida de nuestro intento por responder esta pregunta. Esto es, la conclusión podría ser que hay estándares que no necesitan justificación normativa de su contenido (lo cual es diferente de una justificación de su validez), pero cuyo contenido justifica todos los demás contenidos que son considerados derecho.

En realidad, las preguntas propuestas son bastante cercanas al proyecto del positivismo jurídico. Toda su empresa partió de una simple observación: hay casos paradigmáticos en que la exigencia jurídica parece bastante clara. Por ejemplo, "de acuerdo con el derecho está prohibido matar a la propia esposa si ella no prepara la cena”. El punto es: ¿por qué estamos tan seguros de ello? Si lo estamos, entonces debe haber una forma de conocimiento detrás de la identificación del derecho. Para revelarla, la epistemología jurídica debería identificar cuáles son los hechos que hacen verdadera a dicha proposición acerca del derecho.

En un ambiente inspirado por la evolución de las ciencias naturales, e influenciado por el relativismo ético, la respuesta buscada por los positivistas no podía yacer en el descubrimiento de algún tipo de hechos morales ni en nada que pudiera depender de la evaluación moral del contenido del derecho. La clave era encontrar un "input observacional externo" al derecho: la tesis de las fuentes sociales. Así, habría casos particulares de acciones que son obligatorias/prohibidas/permitidas simplemente porque ese tipo de acción era el objeto de una regla de la autoridad constituida de facto ${ }^{1}$. El contenido de esta regla o de la naturaleza de aquella acción no necesita ser (moralmente, subjetivamente) evaluado.

El siguiente paso fue convertir esta implicancia en una definición de lo que es el derecho. Nosotros no tenemos que hacer lo mismo y podemos restringirnos a usar la idea de que hay una fuente objetivamente identificable de reglas jurídicas que es independiente de la apreciación del contenido de las reglas. ¿Es esto suficiente para erigir

\footnotetext{
${ }^{1}$ No voy a entrar aquí en las complejas discusiones sobre el carácter de hecho o normativo de la regla de reconocimiento o de la Grundnorm. Véase (Himma, 2002) para una discusión introductoria sobre la regla de reconocimiento de Hart. Para la Grundnorm de Kelsen véase (Vernengo, 1960).
} 
un sistema jurídico? En contra de algunos positivistas, de forma preeminente, de Kelsen, creo que no lo es.

Supóngase, por ejemplo, una comunidad nubosa que justifica sus acciones basada en la forma de las nubes. La fuente del derecho es objetivamente identificable: simplemente mírese al cielo. Pero su inteligibilidad parece insuficiente para justificar acciones o decisiones acerca de acciones. Si no hay una instancia clara de corrección o incorrección para alguna forma de nube, dudo que podamos describir tal práctica como una práctica de seguir reglas. Aun si en la comunidad nubosa la mayoría de los ciudadanos entiende que la forma $x$ se corresponde con la incorrección de la acción $y$, no hay un estándar convenido que permita a la mayoría criticar a aquellos que hacen $y$ luego de observar la forma $x$. Si la convergencia de la mayoría es el estándar para la crítica, entonces no es la forma de las nubes sino el comportamiento convergente de la mayoría (o de un grupo) el que constituye la fuente y dicha fuente tiene casos claramente identificables. Así que parece que debe haber no sólo una fuente objetivamente identificable, sino también casos claramente identificables de los estándares provistos por la fuente ${ }^{2}$. Entonces, ¿cuáles podrían ser los criterios para identificar el conjunto de ejemplos objetivos de estándares derivados de la fuente social objetiva? En otras palabras, ¿cuáles son los casos claros que podrían jugar el rol de conjunto básico de evidencias dentro de una teoría coherentista de la interpretación jurídica?

Una sugerencia inicial sería la de considerar claros a aquellos casos que se derivan inmediatamente de los términos de las reglas sin necesidad de interpretación (Marmor, 2005). Esto se corresponde con la clásica idea de clara non sunt interpretanda, propuesta por ejemplo por Leibniz, para quien la claridad sería una cualidad del significado de las reglas y está presente cuando lo captamos inmediatamente. Veamos si esta propuesta funciona:

El Código Penal Brasileño, en su artículo 124, establece que "el aborto está prohibido". Un poco más abajo, otra regla, el artículo 128 dice "está permitido el aborto mediando consentimiento si la vida

${ }^{2}$ Esta era la tesis de Hart. Una versión más reciente de este punto es dada por (Marmor, 2005). Véase una crítica en (Ratti, 2005). 
de la mujer está en peligro o si el embarazo es resultado de abuso sexual". Hace algunos años estas dos reglas fueron el centro de una polémica decisión de la Corte Suprema Brasileña sobre el derecho al aborto de fetos anencefálicos. Aunque hubo discrepancias sobre varios puntos, nunca nadie cuestionó el "hecho lingüístico" de que de acuerdo con el artículo $124^{*}$ el aborto está prohibido si no media riesgo para la madre ni abuso sexual.

¿Cómo es posible ese entendimiento establecido? El lector puede pensar que mi reacción es incómoda, pero nótese que el contenido del artículo $124 *$ no es lo que el artículo 124 dice. En realidad, ;el artículo 124 y el 128 son inconsistentes! Simplemente no lo vemos porque se ponen en práctica mecanismos implícitos de inferencia. Estas inferencias pueden ser captadas por referencia a algunos principios inmemoriales de interpretación jurídica como lex specialis derogat generalis (para eliminar el 124) y exceptio firmat regulam in casibus non exceptis (para introducir el 124*). De aquí que definir "casos claros" como aquellos en que la regla no necesita interpretación parece mal encaminado ${ }^{3}$. Los casos claros pueden de por sí ser el resultado de cambios del sistema normativo a través de la interpretación.

Quizás el adjetivo "claro" es equivocado y lo que se busca como "objetivo" no es la claridad de la palabra en la regla, sino de su instanciación [instantation]. Deberíamos hablar de casos fáciles como aquellos cuya instanciación es fácil. Siguiendo esta línea de pensamiento, otro intento sería el de definir los casos claros como aquellos donde "la decisión puede estar jurídicamente justificada por medio de un argumento puramente deductivo" (MacCormick, 1994). Atria (1999) interpreta esta sugerencia en contraposición a la tesis de Alexy de que un argumento jurídico suficientemente justificado debe contener no sólo una justificación interna (deducción a partir de premisas jurídicas) sino también una justificación externa (una defensa de las premisas normativas basada en la juridicidad, moralidad,

\footnotetext{
${ }^{3}$ No tengo espacio aquí para desarrollar por completo el argumento y me limitaré a indicar contraejemplos que desafían cada tesis. Para una explicación más detallada véase (Maranhão, 2011).
} 
consecuencias sociales, intención legislativa, etc. $)^{4}$. Así, otra lectura de esta propuesta sería que los casos fáciles son aquellos donde no hay necesidad de justificación externa.

Esta sugerencia también parece inadecuada. Permítaseme citar la versión exacta del artículo 128 del Código Penal Brasileño:

Art. 128: El aborto practicado por un médico no será castigado si:

(i) no hay otro medio de salvar la vida de la madre;

(ii) el embarazo es resultado de un abuso sexual y la madre consiente el aborto.

Nadie en la comunidad jurídica brasileña habría osado negar que el texto de arriba signifique que "está permitido el aborto mediando consentimiento si la vida de la mujer está en peligro o si el embarazo es resultado de abuso sexual". Sin embargo no hay una indicación explícita de que las proposiciones (i) y (ii) deban ser leídas disyuntivamente en lugar de conjuntivamente. Tales proposiciones no son mutuamente excluyentes, entonces, ¿qué hace indisputable a la lectura disyuntiva en la comunidad jurídica?

La explicación debe dirigirse hacia los valores protegidos por dichas reglas. La materia "aborto" está estructurada como una oposición entre el derecho del feto a vivir y la libertad de la madre. El art. 124, que en general prohíbe el aborto, claramente reconoce el derecho del feto a vivir por sobre la libertad de la madre. Resultaría difícil dotar de sentido a esta prohibición si el legislador considerara que los fetos no son materia de consideración moral. La referencia al abuso sexual y al

${ }^{4}$ La lectura de Atria está basada en la posición expresada por MacCormick en la primera edición de Legal Reasoning and Legal Theory. En la segunda edición de aquel libro MacCormick ya apunta su desacuerdo con su afirmación anterior: "Ello muestra por qué el razonamiento deductivo a partir de reglas no puede ser un modo autosuficiente y autosustentable de justificación jurídica. Siempre está encapsulado en una red de razonamientos anteriores y ulteriores a partir de principios y valores, aún cuando una visión puramente pragmática revelaría muchas situaciones y casos en que nadie cree que valga la pena el problema de ir más allá de las reglas en pos de propósitos prácticos." (MacCormick, 1994, p. xiii) 
peligro para la vida de la madre sugiere fácilmente otros dos valores relevantes: la dignidad de la mujer y el derecho de la mujer a la vida. Esta hipótesis se ve reforzada cuando uno considera su capacidad explicativa respecto a otras reglas del código penal tales como la prohibición del abuso sexual y el "estado de necesidad" como una condición que excluye la culpabilidad de un delito (la protección de la propia vida justifica el daño a otros y aún hasta el homicidio).

Entonces, resulta razonable explicar la lectura disyuntiva como el mejor esfuerzo para hacer compatible el derecho a vivir del feto con esos otros dos valores implícitamente sostenidos. La fragilidad de la lectura conjuntiva se hace evidente. ¿Qué sentido podríamos extraer de la diferencia entre el valor de la vida de una madre y el valor de la vida de una madre violada, sugerida por la conjunción? Si sólo las madres que han sufrido una violación y cuya vida está en peligro pueden realizar un aborto (y no aquellas cuyas vidas están en peligro, solamente), ¿cómo puede uno explicar el derecho a matar de aquellos que están en estado de necesidad? La lectura disyuntiva, por su parte, provee una armoniosa relación de preferencia entre valores subyacentes (en el caso de abuso sexual, la libertad de la mujer -dado que se requiere su consentimientoen conjunción con su derecho a la dignidad prevalece sobre la vida del feto y, alternativamente, su derecho a vivir prevalece sobre el derecho del feto a vivir).

El ejemplo muestra que un caso de instanciación sencilla (tal como el caso del aborto de una madre que fue abusada sexualmente pero cuya vida no corre peligro) puede involucrar una justificación externa aún cuando no sea explícita en la decisión jurídica. Esto es especialmente cierto cuando el argumento externo es indisputable en la comunidad jurídica. O, como dice Fuller, a veces el propósito subyacente a la regla es tan obvio que perdemos el rastro de su uso en la identificación de su significado o en su instanciación (Fuller, Positivism and fidelity to law).

También vale la pena notar que la inferencia de 124 a 124* no es deductiva y está relacionada con un cambio en el sistema jurídico. Es una transición de un sistema inconsistente $\mathrm{K} 0=\{124,128\}$ a uno consistente $\mathrm{K} 1=\left\{124^{*}, 128\right\}$. En la regulación del aborto todos parecen haber hecho, naturalmente, la misma elección con respecto al conjunto $\mathrm{K} 1=\{124,128\}$. Dos alternativas inmediatas serían: eliminar el art. 124 
o el 128. Éstas se descartan porque son demasiado radicales y menoscaban el arraigado principio de interpretación jurídica de máxima conservación del material jurídico-nihil facile mutandum in solemnibus (Digesto 4, 11.7). En cambio, todos decidimos debilitar o "refinar" el art. 124, cualificando su prohibición por la conjunción de dos factores (ausencia de riesgo para la vida $y$ ausencia de abuso sexual). Hemos elegido K1 porque parecía la mejor alternativa para otorgarle sentido a una legislación razonada.

De este modo, las ideas de "significado establecido del derecho" o de un "caso fácil" no pueden ser capturadas como una cualidad semántica de la formulación normativa ni como una propiedad de la instanciación. Hart ha alertado sobre la sobresimplificación que hay en ver el acuerdo sobre los casos que caen dentro del alcance de una regla como un acuerdo sobre las convenciones lingüísticas compartidas (Hart, 1983: 106). Los casos claros pueden ser el resultado de interpretación que involucre tanto argumentos sobre los objetivos de la regla como sobre valores y elecciones basadas en preferencias entre sistemas jurídicos alternativos. Lo que es característico de ellos es que el cambio resultante de la interpretación o el argumento que defiende externamente las premisas normativas es indisputable dentro de la comunidad jurídica. Entonces podemos decir que casos claros o fáciles son aquellos en que la justificación interna y externa de una regla es indisputable.

Podemos pensar a las situaciones prototípicas de las que emergen los casos claros como aquellas en que una norma válida, objetivamente identificada por su fuente, tiene una formulación normativa clara cuya instanciación al caso entre manos es puramente deductiva. Esto puede proveer un conjunto muy restringido que ciertamente no agota exhaustivamente la "base de evidencias" de la interpretación jurídica. Sin embargo, dado que no es ni condición necesaria ni suficiente de "claridad", la sola aceptación del significado provisto por la convención lingüística sin argumentación externa también implica una elección dentro de un sistema de evaluación en el contexto de interpretación.

Tres puntos de esta discusión parecen de particular importancia. 
Primero, el hecho de que la propia base "preliminar" del derecho implique, de por sí, el apoyo a un sistema de evaluación, da una fuerte indicación de que la construcción de un sistema normativo es de naturaleza coherentista. El input externo dado por las fuentes no puede mantenerse sin al menos una parcial objetividad de su contenido. Y la identificación de este conjunto de instancias objetivas de las reglas derivadas de las fuentes implica el apoyo a un sistema de evaluación. Este sistema de evaluación es el que guía sobre cuáles son las elecciones más razonables por las que arribamos a un conjunto estable de ejemplos corrección e incorrección jurídicas explicado por los significados establecidos de las normas. En otras palabras, esto sugiere un modelo de coherencia de la justificación jurídica. Dentro de un marco coherentista estas proposiciones sobre el derecho son aquellas sobre las que la comunidad jurídica está, de acuerdo con su sistema de evaluación, más confiada de que sean probablemente verdaderas.

Segundo, la cuestión antes indicada de que aún los "casos claros" de juridicidad presuponen relaciones de apoyo con otros contenidos de normas aceptadas, aún en aquellos casos en que se corresponden con la instanciación inmediata de significado claro de una regla objetivamente identificada como basada en las fuentes. Esto sugiere que no hay un input completamente externo de juridicidad cuyo contenido sea independiente de cualquier relación de apoyo con otros contenidos aceptados como derecho. Si esta última afirmación es cierta, entonces nuestros mejores candidatos a cumplir el rol de "proposiciones observacionales" en la interpretación jurídica (los casos claros y significados establecidos) no deberían ser considerados como poseedores de un valor en sí mismos.

Pero de alguna manera sabemos, aún si no podemos establecer criterios claros de identificación, que está este núcleo de representaciones establecidas, que es ciertamente una condición conceptual de la práctica de seguir reglas. En definitiva, y esto es el tercer punto, la estabilidad de este conjunto es alcanzada por un consenso dentro de la comunidad jurídica. Algunos ejemplos de aplicación de las reglas y sus significados son aceptados porque su justificación interna y externa no encuentran objeciones y no pueden ser disputadas. Esto sugiere una relación entre coherencia y argumentación en la 
identificación de la base de un sistema normativo. Esto es, la base está dada por el conjunto de instancias de acciones jurídicamente correctas e incorrectas explicadas por un conjunto de reglas cuyo significado es indisputable dentro del stock de argumentos aceptables (dentro de la comunidad de académicos y operadores jurídicos).

\section{LOS PRINCIPIOS JURÍDICOS COMO EL PRODUCTO DE INFERENCIAS ABDUCTIVAS LOCALES DE LA BASE DE REGLAS}

Como hemos visto más arriba, la coherencia en la interpretación jurídica es de un tipo particular y muy difícil de manejar, dado que no hay claridad sobre cómo identificamos la base misma a ser explicada. Sólo sabemos que existe como un conjunto de reglas basadas en fuentes cuyo significado establecido explica inmediatamente instanciaciones no disputadas. Así que debemos abstraernos de ella y tomar la base como algo "dado" si pretendemos avanzar en la idea de definir una clausura coherentista de un sistema normativo.

Supóngase, entonces, que partimos de un conjunto de reglas basadas en fuentes y uno de instanciaciones claras que son explicadas por esas reglas. Podemos clausurar este conjunto por consecuencias lógicas proveyéndonos de soluciones normativas ulteriores. Pero por supuesto este restringido conjunto no disputado es limitado y no refleja un elemento que ya hemos indicado en los ejemplos que hemos discutido: el recurso a valores y principios jurídicos reflejados [endorsed] por las reglas. La expansión y especificación coherentistas de la base dependen de su relación con valores subyacentes.

La inclusión de valores morales y políticas públicas relacionados con normas establecidas debe ser manejada cuidadosamente, o de otra manera la base misma podría perderse. Aunque los principios son usualmente vistos como justificaciones de las reglas jurídicas, aquí vamos a tomarlos al revés.

Podemos diferenciar la explicación de la justificación como relaciones entre estándares normativos. En el modelo, un estándar normativo está justificado por otro si se deriva lógicamente de éste 
último. Un estándar normativo explica al otro contrafácticamente, esto es, si fuese válido entonces el otro sería derivado deductivamente. En esta perspectiva, la relación de explicación es un paso de una inferencia abductiva, dado aquí en tanto una inferencia a la mejor explicación (Harman, 1965). Siguiendo a Harman, este método de razonamiento tiene básicamente tres pasos: (a) recolección de evidencias, (b) formulación de hipótesis que, de ser ciertas, basados en nuestro conocimiento, explicarían aquellas evidencias y (c) comparación y selección de las hipótesis más explicativas.

Entonces una regla general válida justifica la validez de su instanciación (regla individual) en la medida en que la instanciación sea lógicamente derivada de ella. Pero un principio jurídico (valor moral o política pública) puede ser interpretado como la mejor explicación del propósito legislativo de la regla o de un conjunto de reglas, lo que significa que el principio se deriva lógicamente de la regla aceptada por abducción ${ }^{5}$. Es la aceptación de la base de reglas lo que justifica la aceptación de un principio jurídico (como un estándar jurídicamente válido) en la medida en que este último provee la mejor explicación de la primera.

El vínculo entre las normas y sus objetivos está basado en algún conocimiento anterior sobre las acciones y sus consecuencias generales o lo que implican moralmente. Está relación está bien captada en la descripción de Schauer de las generalizaciones observacionales

\footnotetext{
${ }^{5}$ En las últimas dos décadas el razonamiento abductivo recibió considerable atención de parte del campo de la Inteligencia Artificial y fueron sugeridos muchos modelos para captar su racionalidad. Estos modelos ayudaron a clarificar cómo la conclusión de una inferencia abductiva está garantizada [warranted] por sus evidencias y trasfondo de asunciones. Dado que este método de inferencia incrementa el contenido [is content increasing], una preocupación central es cómo conferir una garantía epistémica a su conclusión (PSILLOS, 2002). Esta preocupación es afrontada con el desarrollo de estándares de comparación de teorías compitiendo entre sí que están altamente informadas por la estética coherentista (la hipótesis ganadora es consistente, la que explica la mayor cantidad de evidencias, la que se adhiere mejor al propio trasfondo de asunciones, la más simple, sin restricciones ad hoc, la más unificada, la más precisa, etc.). Esto vincula la justificación de la abducción en explicaciones causales a las teorías coherentistas sobre la justificación.
} 
subyacentes a las normas (Schauer, 1991). Por ejemplo, una regla que impone un límite de velocidad para los autos está basada en una observación generalizada de que manejar autos más allá de ese límite incrementa los riesgos de accidentes fatales y en la evaluación de que los accidentes fatales son males (la vida humana es un valor que vale la pena reivindicar). Este valor es la explicación de fondo de la regla del límite de velocidad.

La inferencia abductiva es sensible a variaciones en la base de creencias aceptadas. Si ella es expandida, nuevos elementos pueden contradecir explicaciones previamente aceptadas. Así que hay una gran diferencia entre la abducción de un principio a partir de una regla y la abducción a partir de un conjunto de reglas o de toda la base de reglas aceptadas. Y aquí yace un paso decisivo. ¿Debiéramos considerar a la coherencia como una relación entre toda la base y sus principios explicativos (coherencia global) o entre un específico conjunto de reglas relacionado con una acción en particular y sus principios explicativos (coherencia local)?

MacCormick defiende la coherencia como un valor en el derecho basado en dos factores centrales: (a) el universalismo como una exigencia de racionalidad (las reglas basadas en principios generales que son coherentes entre sí son menos arbitrarias) (ii) la inteligibilidad desde la perspectiva de los sujetos normativos (se presupone que la orden jurídica guía a los sujetos a un estado de cosas ideal, de modo que su organización basada en unos pocos principios coherentes desempeña mejor su rol de guía). Su idea de universalismo lleva a una escala de principios en aumento. Los precedentes o reglas son vistos como vehículos para satisfacer principios que a su turno son instancias de principios o metas más generales, llevando así a un conjunto de unos pocos principios del más alto nivel (MacCormick, 2005: 193). La orden jurídica debe entonces ser ajustada y coherente con esos principios de más alto nivel.

Semejante amplitud al universalismo puede llevar la explicación a la trivialidad, o a elecciones arbitrarias del intérprete. La explicación de una amplia base de reglas relacionada con varios temas puede tener dos consecuencias: o exigirá principios que son demasiado generales como para tener poder explicativo alguno, o, peor, habrá que 
sacrificar las reglas de la base que sean incoherentes con principios aceptados.

El segundo problema ya había sido identificado por Savigny, cuya concepción orgánica del derecho tiene paralelismos interesantes con la epistemología jurídica coherentista. Él concebía la relación entre la regla y su propósito como una "concatenación lógica" que lleva desde la regla a su ratio (ratio legis). Y dicha concatenación podría también sostenerse entre motivos, estableciendo una graduación entre reglas, propósitos y otros propósitos más distantes. La coherencia entre altos propósitos, institutos jurídicos, reglas y fuentes de derecho provee el carácter "orgánico" del orden jurídico y su unidad como la expresión del "espíritu del pueblo" (ratio juris). Pero él tenía la clara preocupación de que la aplicación directa de un propósito cuya concatenación fuese muy distante respecto de la regla pueda ser engañosa, dado que diferentes propósitos específicos (de orden inferior), dando una guía contraria, pueden ser sostenidos bajo el mismo propósito general superior (Savigny, 1886: 246-247). Así, la aplicación directa de este propósito superior puede traicionar la guía específica contenida en la "previsión" de la regla. De acuerdo con Savigny, el uso de los propósitos generales del derecho en lugar de los propósitos específicos de la regla sería una modificación oblicua del contenido del derecho que no merece el nombre de interpretación:

Por el contrario, el propósito general de una regla (por ejemplo, la aequitas sobre la que está basada) no puede autorizar una interpretación que corrija una regla, por considerar su expresión inexacta. De hecho, un procedimiento tal no tiene ya el carácter de interpretación, sino el muy diferente carácter de modificación del Derecho, porque al hacerse eso, lo que se investiga no es más cuál será la provisión de la regla, sino qué provisión debería razonablemente contener la regla si el legislador tuviera una idea clara de ella. (Savigny, 1886: 245, traducción libre).

La misma preocupación es expresada por Raz, argumentando que el ideal de coherencia, por ejemplo, perseguido por la teoría del derecho como integridad de Dworkin, transfiere la base de coherencia 
desde el concreto conjunto de reglas a un conjunto de principios articulado de manera abstracta (vinculado con las evaluaciones morales del intérprete). El resultado es que los principios ganan un estatus prioritario en lugar de cumplir un rol explicativo secundario (por ello es que él rechazó que la teoría del derecho como integridad fuera una teoría de la coherencia). Es curioso que tanto Dworkin como Raz defiendan sus visiones basados en el valor de la imparcialidad. El primero argumenta la integridad de los principios hacen al derecho "hablar con una sola voz", estando sobre y por encima de instituciones políticas particulares. (Dworkin, 1986). El segundo, lee la imparcialidad como fidelidad a la incongruencia y peculiaridades de las fuerzas políticas que componen las diferentes ramas del orden jurídico. (Raz, 1994).

No entraré en esta difícil discusión concerniente a la mejor política para la dogmática jurídica, como un vehículo imparcial para entender el derecho. Sólo puedo decir que, desde la perspectiva de nuestro proyecto, los oponentes parecen estar discutiendo sobre la comprensión del derecho como la clausura coherentista de las reglas que estamos (moral o políticamente) justificados para aplicar o como la clausura coherentista de las reglas que de hecho aplicamos.

Mi voto por una inferencia coherentista local de principios jurídicos a partir de reglas jurídicas aceptadas, descansa tanto en la advertencia de Savigny como en la intuición de que la exigencia de coherencia en la descripción de todo el conjunto de normas válidas no refleja nuestra verdadera manera de entender el derecho. Las preguntas prácticas sobre cuál es el derecho son usualmente hechas para saber qué es lo que esto que consideramos derecho válido dice sobre tal acción en éstas y aquéllas circunstancias. Para responderlas, sólo un pequeño subconjunto de reglas basado en fuentes necesita estar activo. Es innecesario e irrazonable exigir una descripción idealizada y comprehensiva de todo el conjunto de reglas válidas. Por ejemplo, ¿cuál es la relevancia de principios relacionados con reglas sobre la interconexión de redes de telecomunicación con respecto a la permisión o prohibición del aborto? ¿En qué sentido deberían las reglas sobre telecomunicaciones y sobre el aborto formar un todo coherente?

Como señala Harman, quienes realmente están en posición de hacer los razonamientos que nos ocupan tienen recursos limitados y no 
tienen la capacidad cognitiva para procesar todo el conjunto de creencias o de mantener un registro de sus justificaciones cuando deducen una conclusión teórica o práctica. (Harman, 1989). Él también propone que una epistemología sensible no debería intentar asumir semejante ideal de completitud para justificar nuestras creencias y nuestras disposiciones a cambiarlas. Lo mismo se aplica al razonamiento jurídico. Dado un caso (real o hipotético) la cuestión jurídica relevante es si una acción en particular es correcta o no. El intérprete entonces selecciona un subconjunto de las reglas provistas por la convención sobre las fuentes autoritativas, que directa o indirectamente (con el auxilio de algunas definiciones legales o doctrinales) se refieran a tal acción (y tal vez a acciones similares o relacionadas con la acción central). Cualquier discusión sobre normas derivadas usualmente se desenvuelve y sólo tiene sentido si se refiere a un subconjunto relevante de la base de reglas aceptadas.

\section{INFERENCIAS DINÁMICAS LOCALES A PARTIR DE LA BASE}

La búsqueda de la justificación de fondo de un conjunto de reglas locales por una inferencia coherentista o abductiva no depende de la afirmación de que la coherencia puede garantizar la verdad de proposiciones jurídicas. La propia práctica de interpretación en la comunidad jurídica es llevada a cabo con la idealización de un legislador racional y coherente. De allí que, en términos de Lipton (Lipton, 2007), la meta no es la explicación más probable (likeliest) de las reglas, porque no estamos buscando la intención legislativa real. Procuramos la mejor (loveliest) explicación, dado que el comportamiento convergente de la comunidad idealiza un legislador cabal que realiza elecciones coherentes (razonadas y consistentes).

Así como en la investigación científica la búsqueda de nuevas teorías o explicaciones es consecuencia de lo que Peirce llamo "observaciones sorprendentes", también en el derecho la apelación a una explicación de fondo de una regla por medio de una "inferencia al mejor propósito legislativo" está provocada por algún tipo de sorpresa dada por un caso que resultó "no predicho" respecto de la base de reglas 
aceptada. Una observación es sorprendente si es una novedad, i.e., si nuestras creencias actuales no explican la observación, o si es una anormalidad, i.e., nuestras creencias nos llevan a una explicación que contradice la observación (ALISEDA, 2000).

\subsection{Novedades}

Podemos tomar como novedades los casos de "lagunas" considerados en un sentido muy amplio que pueda incluir un concepto naïve tal como "no hay una regla relacionada con alguna acción cuyo estatus deóntico deba ser definido y justificado jurídicamente", así como a una laguna normativa, i.e., alguna acción jurídicamente regulada que tiene su estatus deóntico indefinido en un caso relevante, o a una laguna de reconocimiento, esto es, una problema de instanciación de una regla debido a sus términos vagos o ambiguos (véase Alchourrón y Bulygin, 1971, caps. 1 y 6). En todos estos casos de "laguna", parece no haber justificación provista por la base aceptada de reglas para una solución a una descripción particular de una acción.

Para hacerse cargo de estos casos, la base de reglas establecidas debe ser expandida de manera tal que ellos se vuelvan explicables por la simple instanciación de la base. ¿Pero cómo debiera ser expandida? Propongo dos principios guías:

Expansión coherentista (COH): la expansión debiera incorporar estándares o definiciones que son coherentes con la base.

Expansión minimal (MIN): la expansión no debiera exceder lo necesario para justificar el nuevo caso.

La "minimalidad" está basada en la idea de que mientras más cerca nos mantenemos de la base aceptada y establecida, más fuerte será la aceptación del nuevo estándar normativo. Será más probable reunir ambos requerimientos si tenemos éxito en explicar/justificar el estatus deóntico de la acción relevante en la nueva situación mostrando que 
queda explicada por estándares normativos que ya están "implícitamente" reflejados por la base, esto es, si el trasfondo de principios explicativos del subconjunto relevante de la base provee una solución normativa. Por ejemplo, tómese como "novedad" la acción de entrar al parque con una bicicleta, dada la regla "están prohibidos los vehículos en el parque". Si inferimos que la mejor explicación de esta regla (quizás junto con otras reglas relacionadas tales como la permisión de encender la radio en el parque) pasa por la evitación de accidentes, entonces si aceptamos que las bicicletas ofrecen dicho riesgo, la entrada con bicicletas debiera ser prohibida. De estas consideraciones, proponemos un principio derivado:

Expansión conservadora (CON): la expansión de la base no debiera exceder lo que es necesario para justificar la solución normativa derivada de los principios reflejados por la base.

¿Cómo podríamos describir lógicamente tal razonamiento?

Dos formas principales ${ }^{6}$ de representar la inferencia abductiva aparecieron en el campo de la inteligencia artificial. Primero, los modelos de inferencia la ven como una deducción invertida basada en una teoría de fondo donde la inferencia satisface algunas restricciones (tales como la consistencia de las hipótesis con la teoría de respaldo, la minimalidad en la derivación del explananda y la no derivación del explananda de la teoría de fondo por sí misma). Segundo, puede ser representada desde el punto de vista de un proceso epistémico de expansión de nuestro conjunto de creencias.

La discusión anterior naturalmente sugiere el uso de una estructura o marco de revisión de creencias. En el modelo de Pagnucco, por ejemplo, la expansión abductiva resultante de un conjunto $K$ con respecto a una creencia $a$ está dada por una elección entre las extensiones

\footnotetext{
${ }^{6}$ Uno también puede referirse al método de Thagard de medir la coherencia por la satisfacción de restricciones como una herramienta para la inferencia abductiva o coherentista si tomamos una creencia que incrementa el nivel de coherencia de un conjunto de creencias (con respecto a su propia negación) como derivable de dicho conjunto.
} 
mínimas de este conjunto del que deriva $a$. Wassermann y Dias han aplicado el modelo de Pagnucco a conjuntos que no están necesariamente cerrados por una consecuencia lógica. Olsson propone un modelo de coherencia en el que un modelo de revisión de creencias está restringido por un conjunto referencial que el llamó "conjunto de estabilidad" [stability set] (una clase de conjuntos de creencias). La base original de creencias es expandida por una nueva creencia que luego es "consolidada", esto es, es hecha consistente, dado que la elección entre las alternativas consistentes está restringida por el conjunto de estabilidad. Si la nueva creencia ya es consistente con la base, podemos tomar el modelo de Olsson como una expansión abductiva restringida.

Como señala acertadamente Amaya, los modelos de revisión de creencias son demasiado abstractos y no indican cómo debiera alcanzarse el conjunto coherente resultante. Sólo asumen que es obtenido y luego representan el cambio lógico del original al conjunto coherente resultante, estipulando las condiciones mínimas que dicha modificación debería satisfacer. Todo el "trabajo interesante" que es la definición de criterios de elección de la expansión más coherente es abstraído por una "función de elección". Así, los resultados más coherentes son elegidos, pero nada se dice respecto a cómo elige esta función. Por esto es que Amaya se adhiere al modelo de satisfacción de restricciones de Thagard, que es generoso en criterios de medida que proveen una guía para la elección (aún cuando éstos sean infradeterminados y puedan colisionar).

Pero me siento cómodo con mantener una defensiva distancia de la realidad y confiado en que tales empresas abstractas son útiles para clarificar el terreno en el que se funda cualquier elección (por cualquier criterio) de una "mejor explicación coherentista". Prometo ensayar unas cuantas indicaciones de estos criterios, a pesar de que mi mayor preocupación es defender un modelo "conservador" de explicación, tanto en la expansión como en la contracción de la base. Mi plan es delinear una caracterización lógica de lo que llamo una "actitud conservadora".

En el ejemplo de los vehículos en el parque partimos de una base que contiene una regla referida a la acción de entrar en el parque $(e)$. La base $B$ está compuesta por la regla "si Usted está en un vehículo le 
es prohibido entrar en el parque" $\left(\forall_{\mathrm{x}}\left(\mathrm{V}_{\mathrm{x}} \Rightarrow \mathrm{O}_{\mathrm{x}} \sim \mathrm{e}\right)\right.$, donde $O$ es el operador modal para la obligación, $\Rightarrow$ es implicación estricta y los símbolos para el predicado lógico son los usuales con variables para los sujetos normativos. Junto con un conjunto de creencias de fondo establecidas $K$, incluidas oraciones tales como "La Ferrari de John es un vehículo" ( $f_{j} \Rightarrow v_{j}$, usando j como un nombre para el sujeto John) podemos clausurar la base con instanciaciones no disputadas como "está prohibido para John entrar en el parque manejando su Ferrari”" $\left(f_{j} \Rightarrow O_{j} \sim e\right)$.

La base de creencias también contiene información tal como "los vehículos traen riesgo de accidentes en el parque", "la Ferrari de John trae riesgo de accidente el parque", "el Mercedes de Paul trae riesgo de accidente en el parque", etc. Basado en la idea de que el vínculo entre vehículos y riesgo de accidente es una generalización de estas instancias y de que el creador de derecho pretende evitar los accidentes en el parque, uno puede explicar mejor la base $B$ con el principio de que está prohibido entrar en el parque con cualquier cosa que cause riesgo de accidente $\left(\forall_{x}\left(r_{x} \Rightarrow O_{x} \sim e\right)\right)$. Esta regla es parte de la clausura abductiva del conjunto $B$ que es dada por todos los estándares normativos inferidos abductivamente de $B$. Ahora supóngase que nuestra base de creencias aceptadas también incluye la creencia de que las bicicletas traen riesgo de accidentes en el parque (como una generalización de instancias de accidentes en bicicleta), esto es, $\forall_{x}\left(b_{x} \Rightarrow r_{x}\right)$. Entonces la clausura abductiva de $B$, asumiendo $K$, deriva en $\forall_{x}\left(b_{x} \Rightarrow O_{x} \sim e\right)$, i.e., en que basados en las reglas aceptadas está prohibido entrar al parque andando en bicicleta.

El ejemplo anterior es intencionadamente sencillo. No seguiré explorando las complejidades que involucran estas inferencias, tales como los criterios de comparación de explicaciones alternativas y demás creencias de fondo que son exigidas por esta comparación. Otra dificultad proviene del hecho de que estamos tratando con explicaciones de acción, i.e., buscando razones para la actividad creadora de normas. Y no hay una clara línea de separación entre la comprensión de las razones por las que una acción tuvo lugar y la comprensión de qué acción tuvo lugar, porque para identificar una acción a partir de movimientos corporales (evidencia observacional), uno la vuelve 
inteligible justamente por medio de la atribución de un propósito hipotético al agente ${ }^{7}$. Es por ello que nuestras intuiciones pueden confundirse en la atribución de un propósito al creador de derecho al intentar entender lo que una regla significa ${ }^{8}$.

Para una descripción lógica basta con notar que son tres los pasos involucrados: a) una inferencia abductiva que explicita un nuevo estándar dada la base y un conjunto de creencias de fondo acerca de las acciones y sus resultados; b) la expansión de la base por la incorporación de este nuevo estándar; c) una deducción usando el nuevo estándar normativo explícito y el conjunto de creencias de fondo. Permítaseme primero sugerir algunas toscas definiciones preliminares, tomando a Cn como un operador de consecuencias tarskiano9:

Base local. Sea $S$ el conjunto de reglas establecidas, $K$ un conjunto de creencias de fondo y $a$ un tipo de acción. Entonces $\operatorname{Loc}_{k a}(S) \subseteq S$ es el conjunto de reglas relevantes asociadas con el tipo de acción $a$.

La definición de más abajo está planeada para ser aplicada a la noción naïve de laguna que puede ser descripta como $\Delta \alpha \notin C n(S \cup K)$, donde D es cualquier modalidad deóntica. El esquema aquí desarrollado puede ser adaptado para trabajar con las nociones de laguna normativa y de reconocimiento. No especificaré lógicamente qué podría significar "reglas relevantes asociadas con el tipo de acción $\alpha$ ".

Teorías hermenéuticas. Sea $B$ un conjunto de reglas, $K$ un conjunto de creencias de fondo y $a$ un tipo de acción. Entonces definimos el conjunto de $A b_{k a}(B)$ como el conjunto de los conjuntos de principios mínimos que explican $B$ y definen una solución normativa para $a$ basados en $K$, i.e., el conjunto de todo $\mathrm{Y}$, tal que:

\footnotetext{
${ }^{7}$ Véase (von Wright, 1971 y 1983) y (Stoutland, 2009).

${ }^{8}$ Esta confusión estaba en el núcleo de la controversia Hart vs. Fuller. Véase (Hart, 1958) y (Fuller, 1958).

${ }^{9}$ Esto es, un operador de consecuencias deductivas que satisface las condiciones de auto-deducibilidad, idempotencia y compacidad.
} 
(i) $Y \cap B=\varnothing$

(ii) $B \subset C n(Y \mathrm{E} K))$

(iii) $\Delta a \in C n(Y \cup K))$

(iv) $\perp \in C n(Y \cup K)$

(v) No hay un $Y^{\prime} \subset Y$ que satisfaga (i)-(iv)

La mejor teoría hermenéutica $\operatorname{BestA}_{k}(B)$ es una función de maxielección que selecciona un miembro de $A b_{k}(B)^{10}$.

Ahora podemos elegir la expansión abductiva de una base local de reglas.

Extensiones conservadoras. Sea $S$ un conjunto de reglas, $K$ un conjunto de creencias y $a$ un tipo de acción. Entonces definimos el conjunto $\mathrm{CON}_{\mathrm{ka}}(\mathrm{S})$ como el conjunto de reglas mínimo que derivan en la misma solución normativa dada por la mejor teoría hermenéutica para la base local, i.e., el conjunto de todo $\mathrm{X}$ tal que:

(i) $\operatorname{Loc}_{k a}(S) \subseteq X$

(ii) $\Delta a \in \operatorname{Cn}(X \cup K)$ if $\Delta a \in \operatorname{Cn}\left(\operatorname{BestA}_{k}\left(\left(\operatorname{Loc}_{k a}(S)\right)\right)\right.$

(iii) $\perp \notin C n(X \cup S \cup K)$

No hay un $X^{\prime} \subset X$ que satisfaga (i)-(iii)

Nótese que una extensión de la base local, para derivar en la misma solución normativa dada por los principios que refleja, no necesita incorporar uno de esos principios. Puede ser una simple definición normativa como "Debe interpretarse que las bicicletas son vehículos". La expansión conservadora del sistema jurídico es entonces tan sólo la incorporación de una de las extensiones conservadoras al sistema jurídico original.

\footnotetext{
${ }^{10}$ Dado que estamos tratando con conjuntos no necesariamente clausurados por consecuencia lógica no podemos utilizar una función de cumplimiento total o parcial [full meet or parcial meet function], sino una función de maxielección (véase AGM, 1985); de otro modo nada garantiza que el conjunto resultante vaya a explicar la base de reglas.
} 
Expansión conservadora. Sea $S$ un conjunto de reglas y $C h$ una función de maxielección en $\mathrm{CON}_{k a}(\mathrm{~S})$. Entonces la expansión conservadora de $S$ con respecto a una acción $a$ y a un conjunto de creencias de fondo $K$ (notación: $\operatorname{EXCON}_{k a}(\mathrm{~S})$ ) es definida como $\operatorname{EXCON}_{k a}(\mathrm{~S})=C h\left(\mathrm{CON}_{k a}(\mathrm{~S})\right) \cup \mathrm{S}$.

La caracterización lógica del conservadurismo con respecto a las novedades que he propuesto arriba es capturada por la definición de una expansión conservadora. Sus principales puntos son:

a) Los principios reflejados por la base son aquellos que explican mejor la base original de reglas establecidas

b) La modificación de la base de principios establecidos es mínima, i.e., sólo se añade lo necesario para derivar una solución normativa a la laguna identificada

c) La solución provista por la base expandida de reglas es la misma que la provista por los principios reflejados por la base

\subsection{ANORMALIDADES}

Los casos de anormalidad en el campo de la investigación científica son aquellos en que la evidencia contradice la teoría. Esto es, que la teoría científica ofrece una explicación cuya instanciación es inconsistente con una observación dada. En este caso, la teoría debe ser contraída para evitar la explicación equivocada, o la evidencia debe ser rechazada. Dentro del ámbito jurídico, sin embargo, si partimos de una base de reglas establecidas hay poca posibilidad de derogar reglas basados en un caso. Si la base de reglas es consistente y no hay una laguna con respecto a la acción en cuestión, entonces no debería haber tal cosa como una inconsistencia entre la "evidencia" y el conjunto de reglas aceptadas.

No obstante, podemos considerar anormalidades a aquellos casos de derrotabilidad del conjunto de reglas. Esto es, hay un caso definido por algunos factores relevantes donde la solución normativa 
derivada del conjunto de reglas establecidas es inconsistente con la solución normativa derivada del trasfondo de principios explicativos de este conjunto. Schauer llama a estos casos "experiencias recalcitrantes", que son el resultado de la sobre o infra inclusión de la regla. Alchourrón y Bulygin los llaman "lagunas axiológicas".

Alchourrón y Bulygin han remarcado, a la luz de su propia preocupación por trazar una línea precisa entre el discurso descriptivo y el prescriptivo, que las lagunas axiológicas revelan de hecho un movimiento desde un sistema normativo originario a otro sistema con "casos más finos", donde las condiciones de aplicación de sus normas están mejor especificadas (Alchourrón y Bulygin, 1971). Pero ambos autores parecen haber luego suavizado esta visión. Los diferentes sistemas jurídicos en juego en un movimiento de uno original a uno más fino fueron considerados por ambos como "diferentes niveles de análisis" del mismo sistema normativo. Alchourrón identifica tres posibles disposiciones del creador de derecho, considerando a una norma " $q \Rightarrow \mathrm{O} p$ " y a un factor arbitrario $r$ no explícitamente considerado: $r$ es una excepción implícita (el creador de derecho habría rechazado $q \wedge r \Rightarrow \mathrm{O} p$ ); o $r$ es una no-excepción implícita (el creador de derecho habría aceptado $q \wedge r \Rightarrow \mathrm{O} p$ ); o $r$ es indeterminada en tanto excepción (el creador de derecho no tendría ninguna de las disposiciones mencionadas). El conjunto de consecuencias del sistema jurídico permanece sin cambios en los casos de indeterminación y no-excepción implícita. En el caso de excepción implícita, la solución normativa original se desprende del conjunto de consecuencias lógicas. Bulygin y Schauer tienen una visión similar sobre la derrotabilidad, excepto que la indagación se dirige a las "razones subyacentes objetivas" a la regla.

Un ejemplo puede ayudar a identificar cuáles son los pasos en el razonamiento con anormalidades jurídicas. Tomemos el ejemplo del aborto. De acuerdo con el art. 124 del Código Penal Brasileño el aborto está en general prohibido. Los casos de embarazo resultado de violación y de peligro para la vida de la madre son excepciones explícitas. Ahora supóngase un caso de embarazo donde el feto es anencefálico. Una indagación en los propósitos legislativos sugiere que la dignidad de la madre es superior a la vida del feto, puesto que en el caso de la violación, que puede generalizarse a los casos en que se somete a una persona a 
sufrimientos morales o físicos insoportables, el aborto es permitido. Ahora bien, si el conjunto de creencias de fondo incluye la creencia de que llevar adelante un embarazo con un feto diagnosticado con anencefalia implica un sufrimiento insoportable para la madre, entonces la mejor teoría hermenéutica de principios explicativos asociada con el conjunto de creencias de fondo derivaría en que el aborto en el caso de la anencefalia está permitido. Pero como la anencefalia no es un factor relevante nombrado por las reglas del Código Penal Brasileño como excepción, hay un conflicto entre la instanciación directa de las reglas y la instanciación que proveen las razones subyacentes a las reglas.

Para restaurar la coherencia, debería tener lugar una contracción en el conjunto de reglas establecidas. Así que otra vez tenemos dos principios:

Contracción coherentista $(\mathrm{COH})$ : la contracción debería restaurar la consistencia de la base de reglas con respecto a sus principios explicativos subyacentes.

Contracción mínima (MIN): sólo aquellas reglas en la base local que son responsables de la inconsistencia con el conjunto de principios explicativos subyacentes deberían ser eliminadas.

Pero nótese que la identificación de instancias aceptadas de las reglas establecidas puede involucrar elecciones justificadas entre las consecuencias lógicas de las reglas establecidas. Si el sistema normativo está dado por algunas, pero no por todas las consecuencias lógicas de normas explícitas, entonces se atribuye un estatus epistémico distinto a las reglas explícitas en relación a las implícitas o lógicamente derivadas. Así que estamos tratando con bases (no necesariamente clausuradas) y otra vez el concepto de cambio mínimo en el que se fundan los modelos de revisión de creencias (incluido aquí el modelo de coherencia de Olsson) debe ser revisado. El principio de cambio mínimo hace dos afirmaciones para la contracción: (i) uno debería abandonar normas (creencias) sólo al verse forzado a hacerlo; y (ii) para derogar una norma (creencia) del sistema uno debiera eliminar la menor cantidad de normas (creencias) como sea posible para restaurar la consistencia. 
Para ver por qué el principio de cambio mínimo es insuficiente para conjuntos no-clausurados, tómese el conjunto conteniendo sólo el art. 124: "El aborto está prohibido". Como consecuencia tenemos tanto que "El aborto está prohibido si el feto es anencefálico" como "El aborto está prohibido si el feto no es anencefálico". Ahora bien, si los principios explicativos proveen la solución de que "El aborto está permitido si el feto es anencefálico”, entonces sólo una parte del art. 124 es defectuosa.

Si el conjunto de creencias original está lógicamente clausurado y por ende las oraciones derivadas tienen el mismo estatus que cualquier otra dentro del conjunto, entonces el operador de contracción eliminará la oración "El aborto está prohibido si el feto es anencefálico". En consecuencia, la oración original "El aborto está prohibido" será abandonada. Sin embargo, dadas algunas restricciones (véase Maranhão, 2007), la oración "El aborto está prohibido si el feto no es anencefálico” se mantendrá, tal como es deseado.

Ahora bien, si el conjunto de creencias no está clausurado por consecuencias lógicas, el operador de contracción debe eliminar la oración original de que "El aborto está prohibido" para restaurar la consistencia y, en consecuencia, ninguna de sus creencias derivadas será mantenida en el conjunto coherente resultante (consistente con las soluciones provistas por los principios explicativos subyacentes). El principio de minimalidad queda satisfecho en esta irrazonable forma de contracción, dado que la regla original era inconsistente con los principios y era la única regla responsable de dicha inconsistencia. Esto sucede porque el principio de cambio mínimo aplicado a conjuntos noclausurados no tiene una condición para la calidad o adecuación de las reglas derivadas. Como hemos visto, la regla derivada de que "El aborto está prohibido si el feto no es anencefálico” permanece coherente con el conjunto de principios explicativos. Entonces, ¿por qué no debiera ser mantenido?

Otra vez, propongo una actitud conservadora con respecto a la base de reglas establecidas. Así como la expansión debiera ser conservadora en el sentido de que respete principios implícitamente reflejados derivados de las reglas por abducción, la contracción debería ser conservadora en el sentido de que preserve reglas implícitas 
derivadas por deducción. La operación de cambio en cualquier caso debería preservar las reglas con las que estamos comprometidos dada la base de reglas establecidas.

De modo que en lugar de un cambio mínimo, deberíamos usar un principio de conservadurismo más fuerte (Harman, 1989), de acuerdo con el cual uno todavía está justificado en mantener una creencia en ausencia de una razón especial para no hacerlo (si no hay una razón específica para abandonarla). Podemos interpretar este principio como diciendo que deberíamos mantener a las creencias lógicamente derivadas que no han sido específicamente contradichas (véase Maranhão, 2009). Adaptando esta idea a nuestra discusión en epistemología jurídica, esto significa que deberíamos mantener las normas derivadas del conjunto original que no han sido específicamente contradichas por los principios subyacentes.

Contracción conservativa (CON): sólo las reglas derivadas de la base local que sean específicamente responsables de la inconsistencia con el conjunto de principios explicatorios subyacentes deberían ser eliminadas del conjunto de consecuencias de la base.

Un operador de revisión llamado "refinamiento" que cualifica (en vez de eliminar) una de las oraciones del conjunto original ha sido desarrollado en (Maranhão, 2001, 2007, 2009). Usaré letras griegas para referirme a cualquier regla, que puede ser condicional y que provea una solución normativa a la acción bajo escrutinio. Así que vamos a abstraernos de la forma particular de la regla y de la acción referida, dado que sólo estamos interesados en la representación de un conflicto normativo en una condición particular. Entonces, supóngase que la regla $a$ es miembro del conjunto de reglas establecidas $S$ y que $\alpha \in \operatorname{Loc}_{k a}(\mathrm{~S})$. Así tenemos, para cualquier condición arbitraria $b$, tanto $b \Rightarrow \alpha$ como $\sim b \Rightarrow \alpha$. Ahora supóngase que del trasfondo de principios de la base local elegida se deriva una solución conflictiva justamente para esa condición; supóngase, $b \Rightarrow \sim \alpha \in C n\left(\operatorname{BestA}_{b}\left(\operatorname{Loc}_{k a}(S)\right)\right.$. Esto significa que la regla original es derrotada por los principios de fondo relevantes en una determinada condición, que será denominada condición de refutación. 
Entonces, sólo debería descartarse la solución normativa provista por la regla en presencia de la condición de refutación (a saber, $b \Rightarrow a$ ). Si esta condición de refutación está ausente, las soluciones provistas por la regla original deberían mantenerse. Esto significa que la regla original no debería ser eliminada, sino sólo cualificada, de manera de restaurar la coherencia con el trasfondo de principios $(\sim b \Rightarrow a)$.

El operador que llamo "refinamiento interno" (notación \#) satisface el principio de contracción conservadora. Dado un conjunto de reglas $\mathrm{S}$, una regla $\alpha \in \operatorname{Loc}_{k a}(S)$, una función $C n^{*}$ selecciona parte del conjunto de consecuencias no-tautológicas de $\alpha$, i.e. $C n *(\alpha) \subset C n(\alpha) \backslash C n(\varnothing)$ y la función de contracción opera sobre $\operatorname{Loc}_{k a}(S) \cup C n^{*}(\alpha)$. La selección es indicada por la mejor teoría hermenéutica, esto es, si $b \Rightarrow \sim \alpha \in C n\left(\operatorname{BestA}_{b}\left(\operatorname{Loc}_{k a}(S)\right)\right.$, entonces $C n *(\alpha)=\{\sim b \Rightarrow a, b \Rightarrow a\}$. Luego, el operador contraerá sólo la oración específicamente contradicha, tal que $b \Rightarrow a$ no sea parte de las consecuencias del conjunto resultante y la versión cualificada $\sim b \Rightarrow a$ sea incorporada en el conjunto de reglas explícitas establecidas. En otras palabras, dado un sistema normativo $S$, una regla $\alpha \in \operatorname{Loc}_{k a}(S)$, y la elección de una condición $b$ que derrote a $\alpha$, el refinamiento interno de $S$ por $\alpha$ con respecto a $b$ (notación: $\mathrm{S \#}_{b} \alpha$ ) está caracterizado por las siguientes propiedades: ${ }^{11}$

\begin{tabular}{|l|l|}
\hline Corte de raíz [Root-cutting] & $b \Rightarrow \alpha \notin C n\left(\mathrm{~S} \#_{b^{\alpha}}\right)$ \\
\hline Inclusión & $\mathrm{S}_{\mathrm{b} \alpha \subset \mathrm{S} \cup\{\sim b \Rightarrow \alpha, b \Rightarrow \alpha\}}$ \\
\hline $\begin{array}{l}\text { Mantenimiento del núcleo [Core- } \\
\text { retainement] }\end{array}$ & $\begin{array}{l}\text { si } \gamma \in \mathrm{S} \backslash \mathrm{S} \#_{\mathrm{b}} \alpha \text { entonces hay } \\
\Gamma^{\prime} \subset \Gamma \cup\{\sim b \Rightarrow \alpha, b \Rightarrow \alpha\}, \text { tal que } \\
\alpha \notin C n\left(\mathrm{~S}^{\prime}\right) \text { pero } \alpha \in C n\left(\mathrm{~S}^{\prime} \cup \gamma\right)\end{array}$ \\
\hline Preservación & $\sim \beta \Rightarrow \alpha \in \mathrm{S}{ }_{\mathrm{b}} \alpha$ \\
\hline
\end{tabular}

${ }^{11}$ Para más detalles en la construcción de refinamiento y caracterización axiomática, véase (Maranhão 2009). En aquel trabajo, dichos resultados son 
El corte de raíz garantiza que no todas las reglas originalmente derivadas sean válidas, puesto que la regla derivada que fue específicamente atacada por la condición de derrotabilidad ha sido eliminada. La inclusión le permite al operador satisfacer el conservadurismo. El mantenimiento del núcleo es una versión del principio de cambio mínimo aplicado a un conjunto más grande que el original. Dice que sólo deberían ser eliminadas aquellas normas que son de alguna manera responsables de la derivación de la norma específicamente derrotada. La preservación provee la cualificación deseada de la regla original con respecto a la condición de confirmación (que es la negación de la condición de derrotabilidad).

El operador de refinamiento interno simplemente cualifica la regla original, tal que su previsión [detachment] dependa de la condición de confirmación, y por consiguiente la base de reglas asentadas se vuelva coherente con el conjunto de principios explicativos de fondo. El complemento de esto consiste en incorporar a la base de reglas la regla o solución normativa derivada del conjunto de principios reflejados. Esta nueva regla señala [detaches] la solución opuesta en presencia de la condición de refutación. La definición de tal operador es como sigue:

Sea $S$ un conjunto de reglas, $\alpha \in \operatorname{Loc}_{k a}(S)$,

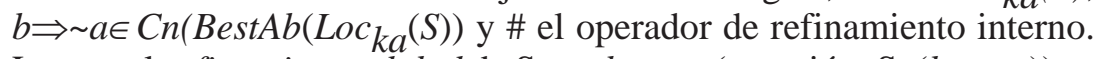
Luego, el refinamiento global de $\mathrm{S}$ por $b \Rightarrow \sim \alpha$ (notación: $\mathrm{S} \bullet(b \Rightarrow \sim \alpha)$ ) es tal que: $\mathrm{S} \bullet(b \Rightarrow \sim \alpha)=\mathrm{S} \#_{\mathrm{b}} \alpha \cup\{b \Rightarrow \sim \alpha\}$.

La caracterización del conservadurismo con respecto a las anormalidades es captada por la operación de refinamiento (interno o

primero dados por cualquier elección $\mathrm{Cn}^{*}$ del conjunto de consecuencias de la oración contraída y luego el refinamiento es sólo un caso especial en el que la elección selecciona sólo una oración y su complementaria (en el sentido allí definido). La construcción es muy abstracta y sus resultados se sostienen para cualquier teoría en cualquier lógica subyacente cuyo condicional satisfaga la descomposicionabilidad [decomposability]: por cualesquiera oraciones a hay oraciones $\mathrm{w}(\alpha)$ and $\mathrm{w}^{\prime}(\alpha)$ tales que (i) $\left\{\mathrm{w}(\alpha), \mathrm{w}^{\prime}(\alpha)\right\} \subseteq \operatorname{Cn}(\alpha)$, y (ii) $\alpha \in$ $\mathrm{Cn}\left(\left\{\mathrm{w}(\alpha), \mathrm{w}^{\prime}(\alpha)\right\}\right)$. Estas propiedades son satisfechas por los condicionales estrictos S5 usados por Alchourrón como la base de su lógica de las proposiciones normativas LNP y también DFT. 
global), que es una forma particular de contracción conservadora. Sus puntos centrales son:

los principios reflejados por la base son aquellos que mejor explican la base original de reglas establecidas

la modificación de la base de reglas establecidas es no sólo mínima sino también conservadora: sólo se remueve aquello necesario para restaurar la coherencia con el conjunto de principios reflejados; las reglas derivadas que no están específicamente en conflicto con aquellos principios son mantenidas, aún si pierden el respaldo que tenían en el conjunto original

la regla en conflicto con el conjunto de principios reflejados en una condición de refutación particular no es eliminada, sino debilitada; se vuelve cualificada y se mantiene, en ausencia de una condición de refutación.

\section{Convergencias o divergencias con Amaya}

Las construcciones anteriores caracterizan lo que yo llamo una actitud conservadora para con la clausura coherentista de la base de reglas establecidas. Cada movimiento es dependiente de la elección del conjunto de principios que mejor explican el conjunto de reglas con respecto a una acción y a un conjunto de creencias de fondo. Sólo son aceptadas adiciones mínimas que sean coherentes con los principios reflejados y en caso de conflicto con sus principios reflejados, la base de reglas establecidas no sufre propiamente derogaciones, sino refinamientos, i.e., algunas de sus reglas ven su condición de aplicación más especificada.

De manera que lo que defiendo es una especie de fidelidad a la base original de reglas establecidas. Aún cuando admito que la identificación de esta base de por sí implica operaciones de cambio y argumentación sobre la coherencia, una vez que la base es establecida, sólo muy pocas modificaciones son aceptadas y están justificadas, si están de acuerdo con principios reflejados por la base misma. A partir de este punto, habría modificaciones sólo muy discretas y conservadoras, bajo la forma de expansiones conservadoras y refinamiento (que es una 
contracción conservadora). Amaya, sin embargo, parece sugerir que las expansiones y contracciones son herramientas relevantes para restaurar la coherencia. Mi preocupación yace en los costes de obtener poder explicativo dentro de una teoría de la interpretación jurídica. Podemos construir una interpretación coherente de una base de reglas establecidas e instanciaciones aceptadas, con gran poder explicativo, simplemente con eliminar aquello que le es contrario e incluir lo que es convergente a un conjunto de principios jurídicos elegidos ${ }^{12}$. El camino de la coherencia no debiera yacer en los principios explicativos, sino en la base misma a ser explicada.

Ambos operadores (de expansión conservadora y de refinamiento) dejan dos aspectos importantes sin definir y abiertos para la intervención de un agente inteligente:

(i) ¿Cuáles son los criterios para identificar, dentro del conjunto de reglas establecidas, aquellas que son las "relevantes" respecto de una determinada acción?

Aquí estoy de acuerdo con Amaya en que el contexto juega un rol. Por ejemplo, el grado de "importancia" del caso puede ampliar el conjunto de reglas relevantes, con efectos directos sobre los principios explicativos y por consiguiente sobre la expansión resultante de la base de reglas. Creo, no obstante, que Amaya le asigna a las consideraciones contextuales un rol más amplio que el de mera selección de la base de reglas. De modo que la construcción aquí propuesta está también vinculada a una epistemología jurídica coherentista local y contextual. Nótese que aunque el contexto tiene aquí su rol limitado al de ser un paso en la epistemología coherentista, este paso está en la base misma de la construcción y es decisivo para su resultado.

\footnotetext{
${ }^{12}$ Una apreciación similar es hecha por Lehrer cuando trata sobre la simplicidad como un criterio de coherencia: "Si buscamos tanto simplicidad como coherencia, tendremos el más fuerte de los motivos para rechazar afirmaciones observacionales con el propósito de reducir lo que necesita ser explicado". Lehrer también rechaza el conservadurismo de Harman basado en la idea de que "el derrocamiento de las opiniones aceptadas y los dictados del sentido común son a menudo esenciales para el avance epistémico". Creo que esa crítica no se aplicaría a la idea de una base de reglas establecidas e instanciaciones, que son esenciales para la identificación de un sistema jurídico.
} 
(ii) ¿Cuáles son los criterios con que guiar las dos funciones de maxielección que son usadas en la construcción de sendos operadores? Primero, ¿cómo elegir la mejor teoría hermenéutica de principios que expliquen la base local (este conjunto guiará tanto la elección de la condición de refutación para el refinamiento como la solución a ser lograda por la expansión)? Segundo, ¿cómo elegir la mejor expansión mínima de reglas establecidas?

Otra vez estoy de acuerdo con Amaya en que los modelos de satisfacción de restricciones pueden ser herramientas eficientes y plausibles para guiar semejante selección. Y a pesar de que puede haber casos muy claros que determinen una elección, puede haber varios casos donde los criterios pueden colisionar y más de una solución aparezca igualmente plausible. También sigo la sugerencia de Amaya de que lo mejor que podemos hacer es exigirle responsabilidad epistémica al agente. En definitiva, propongo que una solución normativa a una novedad jurídica o anormalidad está epistémicamente justificada si el agente es responsable o virtuoso en la evaluación de los criterios de coherencia en sus maxielecciones en la expansión conservadora o refinamiento de la base establecida de reglas. Mi diferencia (o no) con Amaya es que yo sostengo una actitud conservadora, en el sentido aquí definido, como un presupuesto de la epistemología jurídica coherentista. Esto es, que ser conservador queda fuera de discusión en cualquier teoría de las virtudes epistémicas de un agente jurídico. 


\section{Bibliografía}

Alchourrón, C. (1996) “On Law and Logic”, Ratio Juris, 9, pp. 331-48. Alchourrón, C., Gärdenfors, P. and Makinson, D. (AGM, 1985), "On the logic of theory change", Journal of Symbolic Logic, 50(2), pp. 510-530.

Aliseda, A. (2000) "Abduction as epistemic change: a Peircean model in artificial intelligence". Flach, Peter A., and Antonis C. Kakas, Editors, Abduction and Induction: Essays on Their Relation and Integration. Dordrecht and Boston: Kluwer Academic Publishers.

Amaya, A. (2006) An Inquiry into the nature of coherence and its role in legal argument, phD. thesis at European University Institute.

Atria, F. (1999) "Del Derecho y el Razonamiento Jurídico", Doxa. Cuadernos de Filosofía del Derecho, $\mathrm{n}^{\circ} 22$.

Bulygin, E. (2005) "En Defensa de El Dorado. Respuesta a Fernando Atria", in Atria et. al. Lagunas en el Derecho. Marcial Pons, Madrid.

Dworkin, R. (1986) Law's Empire, Harvard University Press.

Fuller, Lon L. (1958) "Positivism and fidelity to law: a reply to professor Hart". Harvard law review, v. 71, n. 4: The Harvard Law Review Association.

Harman, G. (1965) "The inference to the best explanation", Philosophical Review, 74, 88-95.

Harman, G. (1989) Change in view: principles of reasoning, MIT Press.

Himma, K. E. (2002) "Inclusive legal positivism". The Oxford Handbook of Jurisprudence \& Philosophy of Law. Oxford: Oxford University Press.

Josephson, J. and Josephson, S. (1984) Abductive Inference: Computation, Philosophy, and Technology. Cambridge, U.K.: Cambridge University Press. 
Lehrer, K. (2000) Theory of Knowledge, Dimensions of Philosophy

Series, Westview Press, 2a. ed.

Levi, I. (2000) "Inductive Expansion and Nonmonotonic Reasoning", in Frontiers in Belief Revision, ed. by Williams, M-A and Rott, H. 7-56. Dordrecht, Kluwer.

Lipton, P. (2004) Inference to the Best explanation, 2a. ed. London and New York: Routledge.

MacCormick, N. (1994) Legal Reasoning and Legal Theory, Clarendon Press, Oxford, reimpressão de 1994 com novo prefácio.

MacCormick, N. (2005) Rhetoric and the Rule of Law. A Theory of Legal Reasoning. Oxford: Oxford University Press, 2005.

Maranhão, J.S.A. (2001) "Some operators for refinement of normative systems". In, Proceedings of the JURIX 2001: The Fourteenth Anual Conference on Legal Knowledge and Information Systems.

Maranhão, J.S.A. (2007) "Refining Beliefs", in J-Y Béziau, A. CostaLeite (eds.), Perspectives on Universal Logic, 335-349, Monza/Italy: Polimetrica International Scientific Publisher.

Maranhão, J.S.A. (2009) "Conservative Contraction", in Carnielli, W., D'Ottaviano, I., Coniglio, M. (eds.) The Many Sides of Logic, series "Studies in Logic", King's College Publications.

Maranhão, J.S.A. (2011) Inclusivismo Lógico: uma contribuição à metodologia jurídica. Tese apresentada na Universidade de São Paulo para obtenção de título de livre-docência, a ser publicada por Marcial Pons.

Marmor, A. (2005) Interpretation and Legal Theory. Oregon: Hart Publishing, rev. 2a. ed.

Olsson, E. (1997) Coherence: studies in epistemology and belief revision, Comprehensive summaries of Uppsala Dissertations, Uppsala.

Pagnucco, M. (1996) The Role of Abductive Reasoning Within the Process of Belief Revision, Phd. Thesis, Department of Computer Science, University of Sydney, 1996. 
Psillos, S. (2002) "Simply the Best: a Case for Abduction", in Kakas, A. (et. al) Computational Logic, LNAI 2408, Springer, Heidelberg, pp. 605-625.

Raz, J. (1994) “The value of coherence", in Ethics in the Public Domain, Oxford, Oxford University Press.

Ratti, G. B. (2008) "The consequences of defeasibility". Analisi e diritto. Torino: Giappichelli, 2008.

Reid, T. (1895) Essays on the Active Powers of man, from The Works of Thomas Reid, D.D., ed. Sir William Hamilton, Edinburgh, James Thin, 617.

Savigny, F.K. (1886) Sistema del Diritto Romano attuale. Translated by Vittorio Scialoja. Torino: Unione Tipografico Editrice.

Schauer, F. (1991) Playing by the Rules: A Philosophical Examination of Rule Based Decision-Making in Law and in Life, Clarendon Press, Oxford.

Stoutland, F. (2009) "Von Wright's Compatibilism”, in Philosophical Probing, Essays on Von Wright's Later Work, F. Stoutland, ed. Automatic Press.

Thagard, P. (2000) Coherence in Thought and Action, MIT Press, Cambrigde.

Vernengo, R. J. (1960) "La función sistemática de la norma fundamental". Revista Jurídica de la Universidad de Buenos Aires. [S.I.: s.n.], 1960.

von Wiright, G.H. (1971) Explanation and Understanding, Routledge and Keagan Paul.

von Wright, G.H. Wassermann, R. and Dias, W (2001). “Abductive Expansion of Belief Bases", Proceedings of the IJCAI Workshop on Abductive Reasoning, Seattle, August, 2001. 